ISSN 1991-8631

Original Paper

http://indexmedicus.afro.who.int

\title{
Contribution à la connaissance des produits forestiers non ligneux du parc national de Kalamaloué, Extrême-Nord Cameroun : les plantes alimentaires
}

\author{
J.L. BETTI ${ }^{1 *}$ et S.R. MEBERE YEMEFA'A ${ }^{2}$ \\ ${ }^{l}$ Département de Biologie des Organismes Végétaux, Faculté des Science, Université de Douala, Cameroun, \\ BP 24157 Douala, Tel : 00 (237) 77303272 ; \\ ${ }^{2}$ NATURE + Asbl, Cameroun, BP 4558 Yaoundé, Tel:(+237) 97497917,E-mail: yemefaa@yahoo.fr \\ *Auteur correspondant, E-mail: lagardebetti@yahoo.fr
}

\section{RESUME}

L'enquête ethnobotanique conduite sur les produits forestiers non ligneux du $1^{\text {er }}$ avril au 30 septembre 2005 dans les ménages des villages basés autour du parc national de Kalamaloué a permis de recenser 26 espèces végétales utilisées comme plantes alimentaires par les populations Arabes choa, Bornoa, Kotoko, et Mousgoum. Ces espèces sont distribuées dans 20 genres, 15 familles et interviennent dans 49 recettes alimentaires différentes. Les fruits, graines et feuilles sont les organes végétaux les plus cités, tandis que les amuse-gueule sont les modes de consommation des plantes les plus mentionnés. Les espèces telles que Balanites aegyptiaca, Tamarindus indica, Boscia senegalensis, Diospyros mespiliformis et Ziziphus mauritiana apparaissent comme les plantes les plus utilisées comme alimentaires. Les fruits produits par les espèces comme $B$. senegalensis et $Z$. mauritiana permettent aux communautés de survivre même pendant les périodes de disette. Les espèces indiquées à Kalamaloué sont également mentionnées pour les mêmes usages dans d'autres pays africains. L'administration en charge de la gestion des parcs devrait tenir compte de l'importance des produits forestiers non ligneux de nature végétale dans la vie des populations riveraines du parc national de Kalamaoué pour éviter des conflits.

(C) 2011 International Formulae Group. All rights reserved.

Mots clés : Plantes alimentaires, parc national de Kalamaloué, légume, amuse-gueule, épice, plat principal.

\section{INTRODUCTION}

La création des aires protégées au Cameroun cadre avec la volonté du gouvernement de préserver des exemples des principaux écosystèmes afin de maintenir la diversité biologique. L'objectif à terme du Gouvernement du Cameroun en matière de conservation de la biodiversité est de convertir $30 \%$ du territoire national en aires protégées. Ces aires protégées comprennent les parcs nationaux, les réserves naturelles, les jardins zoologiques,... (Tieguhong et Betti, 2008). Un parc national se définit comme: «un périmètre d'un seul tenant dont la conservation de la faune, de la flore, du sol, de l'atmosphère, des eaux, et en général du milieu naturel, présente un intérêt spécial qu'il importe de préserver contre tout effort de dégradation naturelle et de soustraire à toute intervention susceptible d'en altérer l'aspect, la composition et l'évolution. » (MINEF, 1994, 1996). A ce jour, le Cameroun dispose 
de 19 parcs nationaux. Huit seulement de ces parcs (42\%) disposent d'un plan d'aménagement.

La région de l'Extrême Nord est une région connue pour ses contraintes particulièrement sévères. Ces contraintes touchent à la fois aux rigueurs du climat et à la gravité du risque de désertification. Elles concernent également des handicapes sociaux comme la pauvreté d'une population de plus en plus nombreuse (Tersigue et Becker, 1997), et qui dépend étroitement des ressources naturelles pour satisfaire ses besoins primaires de survie. La région de l'Extrême Nord regorge trois parcs nationaux : Mosogo gokoro (1400 ha) situé dans le département du Mayo Tsanaga, kalamaloué (4 500 ha) et Waza (170 000 ha), situés dans le département du Logone et Chari.

Le parc national de Kalamaloué a été crée en 1972 et ne dispose pas encore d'un plan d'aménagement pour sa gestion durable. C'est un exemple d'aire protégée problématique, où la population riveraine exerce une pression forte sur les ressources naturelles. Les menaces principales sont le braconnage ou chasse commerciale, le pacage des animaux domestiques (bœufs, moutons, chèvres), les feux de brousse liés aux stratégies pastorales, et la récolte du bois de feu.

Les produits forestiers non ligneux (PFNL) pourraient se référer à des biens et services marchands ou de subsistance destinés à la consommation humaine ou industrielle et dérivés des ressources et de la biomasse renouvelables de la forêt, permettant d'augmenter les revenus des ménages ruraux et de créer des emplois. Ces produits comprennent l'utilisation des plantes à des fins diverses : aliments, boissons, fourrages, combustible et médicament; animaux, oiseaux et poissons pour l'alimentation, les fourrures et les plumes; et services de la terre pour la conservation et les loisirs (FAO, 1995a). Lorsqu'on parle de parc national au Cameroun, on pense tout d'abord à la chasse ou de manière générale aux productions secondaires de nature animale. Les produits forestiers non ligneux de nature végétale encore appelés «récoltes cachées » ont souvent été négligés. Il est pourtant démontré qu'en Afrique, les PFNL sont d'une très grande utilité pour les populations rurales et une catégorie des populations urbaines à plusieurs titres: ils constituent une source de revenus non négligeable, contribuent à la sécurité alimentaire et sont pourvoyeurs de nombreux produits indispensables à l'alimentation, la santé, bref au bien être de cette catégorie sociale défavorisée. A ce titre, ils constituent un instrument efficace de lutte contre la pauvreté (Ndoye et Ruiz-Perez, 1999). Jusqu'ici, aucune recherche n'a été publiée sur les PFNL de nature végétale du parc national de Kalamaloué. La connaissance de ces ressources est importante à un triple point de vue: les résultats peuvent être exploités dans le processus de rédaction du plan d'aménagement du parc en vue de l'établissement des systèmes de gestion participative durable des ressources du parc; les résultats peuvent permettre ou alors amorcer une meilleure valorisation de ces produits qui peuvent contribuer à la sécurité alimentaire et à la santé des populations riveraines; et enfin les résultats peuvent stimuler une valorisation de ces produits qui peuvent constituer une ressource génératrice des revenus complémentaires et donc une alternative à la chasse identifiée comme l'une des menaces principales du parc.

La présente étude analyse l'usage traditionnel des plantes alimentaires récoltés dans le parc national de Kalamaloué.

\section{MATERIEL ET METHODES Milieu}

Le parc national de Kalamaloué est situé dans la région de l'Extrême-Nord Cameroun, dans le département du Logone et Chari et plus précisément dans l'arrondissement de Kousséri. La ville de Kousséri est la dernière ville du Cameroun qui fait frontière avec la République du Tchad. Le 
parc couvre une superficie de 4500 ha et s'étire entre le $12^{\circ} 05^{\prime}$ et le $12^{\circ} 1 \mathrm{I}$ ' de latitude Nord et entre 1e14 $49^{\prime}$ et le $14^{\circ} 90^{\prime}$ de longitude Est. La Parc est limité au Nord-Est par le fleuve Logone et Chari qui sert de frontière entre le Tchad et le Cameroun; au Nord par le fleuve Serbewel, extension du Logone qui prend vie près de Fadjé et tarissable en saison sèche; à l'Est par les villages Marako et Seiba situés à $10 \mathrm{Km}$ de Kousséri ; à l'Ouest par Maltam situé à $25 \mathrm{~km}$ de Kousséri; au Sud par la route bitumée Maltam-Kousséri qui est une partie de la route internationale reliant Kousséri à Maroua (Chef-lieu de la région de l'Extrême-Nord) et Kousséri à Maïduguri (Borno State, Nigeria).

Le climat du parc national de Kalamaloué est de type soudano-sahélien à tendance sahélienne très marquée. Il se caractérise par une longue saison sèche (octobre à mai) et une courte saison de pluie (juin à septembre). Les précipitations annuelles sont faibles et irrégulières. Elles varient entre $230 \mathrm{~mm}$ et $700 \mathrm{~mm}$ avec une moyenne d'environ $529 \mathrm{~mm}$. La température du Parc varie entre $21,7{ }^{\circ} \mathrm{C}$ et $35,7{ }^{\circ} \mathrm{C}$ avec une moyenne d'environ $28,4{ }^{\circ} \mathrm{C}$. Elle peut atteindre des extrêmes de $44{ }^{\circ} \mathrm{C}$ (Letouzey, 1968).

La population est constituée essentiellement de Bornoa, Kotoko, d'Arabe Choa et de Mousgoum. On note aussi la présence de quelques tribus minoritaires venues du Tchad ou du Nigeria, et aussi des Massa en provenance du Mayo Danay (Cameroun).

L'agriculture, l'élevage et la pêche sont les principales activités pratiquées dans la région. Les Kotoko ont pour principales activités l'agriculture et la pêche. Pour ce qui est des Arabes Choa, ils sont en majorité des agriculteurs et des éleveurs. Les Mousgoum sont des pêcheurs et pratiquent l'agriculture comme activité secondaire.

\section{Méthode}

La méthode d'enquête utilisée est celle dite «méthode ethnobotanique d'usage populaire ». Cette méthode consiste à interroger les villageois sur l'usage populaire des plantes utiles. L'enquête a été réalisée du $1^{\mathrm{er}}$ avril au 30 septembre 2005 dans les ménages des villages riverains du parc national de Kalamaloué. Dans chaque ménage, les informations pouvaient être recueillies chez quiconque qui était susceptible de fournir des informations utiles et originales sur l'usage populaire des plantes alimentaires. Pour chaque espèce citée, nous avons relevé l'organe végétal utilisé et le mode de préparation ou l'utilisation. Celle-ci pouvait être l'amuse-gueule, le légume, le plat principal, l'épice ou condiment ou alors le thé. Les amuse-gueule sont des plantes qui se consomment soit directement, soit après transformation en dehors du repas principal. Les légumes sont des plantes potagères dont les organes (feuilles, fruits, graines, ...) sont utilisés pour la confection des plats d'accompagnement. Le plat principal constitue l'aliment de base du repas des ménages de la zone indiquée. L'épice est une substance aromatique qui sert à assaisonner les mets. Pour le thé, l'organe végétal est utilisé en décoction.

L'identification des espèces citées comme alimentaires a été facilitée sur le terrain grâce à l'aide de Monsieur NDONG ATCHIKE, enseignant et Chef de l'Herbarium de l'Ecole de Faune de Garoua, Nord Cameroun. Les confirmations des noms des plantes ont été faites en utilisant les documents de Arbonnier (2000) et Souane (1990). Tous les specimens botaniques collectés ont été déposés à l'Herbarium de l'Ecole de Faune de Garoua.

\section{RESULTATS}

Quarante ménages distribués dans quatre groupes ethniques à savoir les Arabes choa, les Bornoa, les Kotoko, et les Mousgoum ont fourni des informations sur l'usage populaire des plantes alimentaires du parc national de Kalamaloué. Un total de 268 citations faisant intervenir 26 espèces végétales dans 49 recettes indiquées comme alimentaires a été relevé. Ces citations sont distribuées comme suit dans les quatre groupes ethniques rencontrés: Arabes choa (161 citations ; 20 espèces végétales), Bornoa 
(38; 14), Kotoko (35; 15), Mousgoum (34 ; 21). Le Tableau 1 présente les 268 citations des plantes alimentaires recensées dans les différents groupes ethniques interrogés.

Les 26 espèces recensées sont distribuées dans 20 genres et 15 familles (Tableau 2). Les cinq espèces végétales les plus largement citées comme alimentaires sont: Balanites aegyptiaca ou dattier du désert (16\% de citations), Tamarindus indica ou tamarinier (12,6\%), Boscia senegalensis $(7,4 \%)$, Diospyros mespiliformis ou ébène de savane $(6,7 \%)$, et Ziziphus mauritiana $(6,3 \%)$. Ces espèces sont indiquées dans au moins trois groupes ethniques.

Les Capparaceae ( 5 espèces et 63 citations) et les Mimosaceae (3 espèces et 11 citations) sont les deux familles les plus représentées de même que le genre Acacia avec trois espèces. L'importance de la famille des Capparaceae ne s'explique pas seulement par sa grande taille (5 espèces). Les espèces qui la composent font partie des plantes largement citées notamment Boscia senegalensis (20 citations), Capparis fascicularis (14), Capparis sepiarata (14) et Crateva adansonii (14).

Quarante neuf recettes ont été citées par les populations du parc national de Kalamaloué. Elles sont distribuées comme suit dans les quatre groupes ethniques : Arabes choa (38 recettes), Bornoa (23), Kotoko (23) et Mousgoum (21).

Six types d'organes végétaux ont été cités: les bulbes, les feuilles, les fleurs, les fruits, les graines, les gommes et les tiges (Figure 1). Les fruits (53\% de citations) sont les organes végétaux les plus cités comme alimentaires, suivis des feuilles $(20,5 \%)$ et des graines $(20,1 \%)$.

L'importance des citations des modes d'utilisation (consommation) des plantes alimentaires indiquées est illustrée dans la Figure 2. Les organes végétaux prélevés sont généralement consommés comme amusegueule ou fruits de bouche $(52,6 \%)$.

L'usage comme amuse-gueule (plante qui se consomme en dehors du repas) est très indiqué avec une vingtaine d'espèces végétales. Il s'agit des fruitiers sauvages dont on consomme les fruits ou graines comme Balanites aegyptiaca (graine et fruit), Diospyros mespiliformis (fruit), Ziziphus mauritiana (fruit), des gommiers ou espèces du genre Acacia (3 espèces), et d'un tubercule, celui de Nymphaea lotus. Les amuse-gueule sont généralement consommés sans aucune préparation.

Les légumes (plantes potagères dont on consomme les feuilles, les fruits, les graines, les racines, ...) constituent le deuxième mode d'utilisation le plus indiqué par les populations du parc national de Kalamaloué, avec $19,4 \%$ des citations. Les légumes sont cités pour 11 espèces végétales dont les plus indiquées sont: Celtis integrifolia (11 citations), Balanistes aegyptiaca (9), Crateva adansonii (7), Capparis fascicularis (6), et Capparis sepiaria (6). Les feuilles de $C$. integrifolia sont généralement consommées à l'état frais ou sec découpé en petites tranches. Les feuilles séchées sont généralement écrasées et conservées sous forme de poudre. C'est dans ce dernier état qu'elles sont conservées. Le nom de la plante est en général celui que l'on donne au mets préparé à base de la plante. Ainsi par exemple, à l'état frais, sec ou sous forme de poudre, la sauce à base des feuilles de Corchorus tridens est appelée Moulohié en Arabe choa. La pulpe de fruits de Boscia senegalensis sert comme farine de bouillie au lait de vache. Avant leur utilisation, ces espèces de la famille des Capparaceae sont d'abord trempées dans l'eau pendant plusieurs jours. Elles peuvent être ensuite préparées et séchées ou directement séchées. C'est dans ce dernier état qu'elles sont utilisées dans la préparation des sauces locales. On les utilise surtout en période de disette.

Les épices ou condiments sont le troisième mode d'utilisation/consommation des plantes alimentaires du parc national de Kalamaloué avec 14,6\% de citations. Quatre espèces sont mentionnées comme condiments à savoir: Boscia senegalensis, capparis fascicularis, Capparis sepiaria, et Tamarindus indica. Le genre Capparis est utilisé comme épice de sauce tandis que Tamarindus est indiqué comme épice de 
bouillie. En absence de son fruit, les feuilles de Tamarindus peuvent être utilisées. Il n'existe aucune transformation particulière à apporter à l'organe végétal avant son utilisation comme épice ou condiment. Selon les populations locales, $T$. indica est utilisée comme substitut du lait dans la bouillie de maïs, de riz ou de mil ou simplement comme apéritif.

Le plat principal est indiqué dans $13,1 \%$ des usages avec 5 espèces végétales à savoir: Panicum pansum, Echinochloa colona, Oryza barthii, Nymphaea lotus,
Cadaba farinosa. Pour toutes ces espèces, l'organe végétal récolté est séché, décortiqué, écrasé et préparé sous forme de boule de couscous. Il faut tout de même relever que la graine de Boscia senegalensis est toxique, de même que les feuilles de Cadaba farinosa. Ces organes sont préalablement trempés pendant plusieurs jours dans de l'eau. En période de disette ou période de famine, la famille des Capparaceae est particulièrement utilisée comme plats principaux. En période hors disette, ce sont les espèces de la famille des Poaceae qui sont très utilisées.



Figure 1 : Importance relative des citations des organes végétaux comme alimentaires dans le parc National de Kalamaloué.

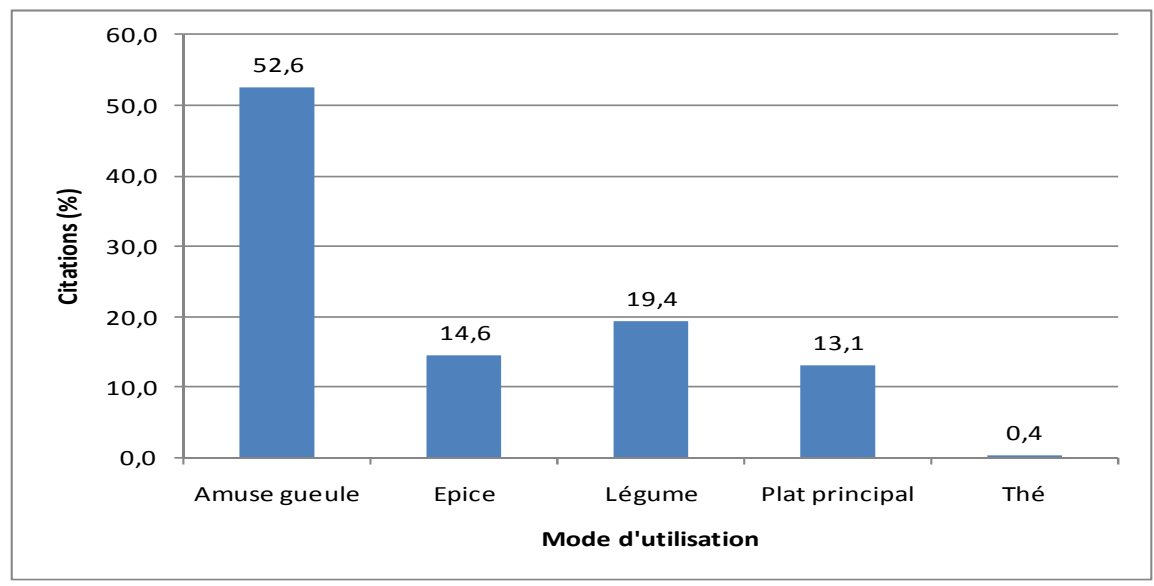

Figure 2 : Importance relative des citations des modes d'utilisation ou de consommation des plantes alimentaires dans le parc National de Kalamaloué. 


\section{J.L. BETTI et S.R. MEBERE YEMEFA'A / Int. J. Biol. Chem. Sci. 5(1): 291-303, 2011}

Tableau 1 : Distribution des usages des plantes alimentaires dans les différents groupes ethniques. Les chiffres dans les groupes ethniques traduisent le nombre de personnes (citations) ayant mentionné la recette indiquée.

\begin{tabular}{|c|c|c|c|c|c|c|c|c|}
\hline Espèce végétale & Organe végétal & Utilisation & Recette & Ar & Bo & Ko & Mo & Total citations \\
\hline Acacia erythrocalyx & Gomme & Amuse-gueule & $\operatorname{Rec} 1$ & 4 & & & & 4 \\
\hline Acacia seyal & Gomme & Amuse-gueule & $\operatorname{Rec} 2$ & 5 & & & & 5 \\
\hline Acacia sieberiana & Gomme & Amuse-gueule & $\operatorname{Rec} 3$ & 2 & & & & 2 \\
\hline Adansonia digitata & Feuille & Légume & $\operatorname{Rec} 4$ & 2 & & 3 & & 5 \\
\hline Adansonia digitata & Fruit & Amuse-gueule & $\operatorname{Rec} 5$ & 3 & & 2 & & 5 \\
\hline Balanites aegyptiaca & Feuille & Légume & $\operatorname{Rec} 6$ & 4 & 1 & 1 & 3 & 9 \\
\hline Balanites aegyptiaca & Fruit & Amuse-gueule & $\operatorname{Rec} 7$ & 11 & 3 & 4 & 3 & 21 \\
\hline Balanites aegyptiaca & Graine & Amuse-gueule & $\operatorname{Rec} 8$ & 7 & 3 & 2 & 1 & 13 \\
\hline Boscia senegalensis & Fruit & Amuse-gueule & $\operatorname{Rec} 9$ & 9 & 2 & & 1 & 12 \\
\hline Boscia senegalensis & Fruit & Epice & $\operatorname{Rec} 10$ & 2 & & & & 2 \\
\hline Boscia senegalensis & Graine & Epice & $\operatorname{Rec} 11$ & 5 & 1 & & & 6 \\
\hline Cadaba farinosa & Feuille & Plat principal & $\operatorname{Rec} 12$ & & & 1 & & 1 \\
\hline Calotropis procera & Fruit & Amuse-gueule & $\operatorname{Rec} 13$ & & & 1 & 1 & 2 \\
\hline Capparis fascicularis & Feuille & Légume & $\operatorname{Rec} 14$ & 1 & & & & 1 \\
\hline Capparis fascicularis & Fruit & Amuse-gueule & $\operatorname{Rec} 15$ & 3 & 1 & 1 & 1 & 6 \\
\hline Capparis fascicularis & Fruit & Epice & $\operatorname{Rec} 16$ & 1 & 1 & & & 2 \\
\hline Capparis fascicularis & Fruit & Légume & $\operatorname{Rec} 17$ & 3 & & & 1 & 4 \\
\hline Capparis fascicularis & Graine & Légume & $\operatorname{Rec} 18$ & 1 & & & & 1 \\
\hline Capparis sepiaria & Feuille & Légume & $\operatorname{Rec} 19$ & 1 & & & & 1 \\
\hline
\end{tabular}


J.L. BETTI et S.R. MEBERE YEMEFA'A / Int. J. Biol. Chem. Sci. 5(1): 291-303, 2011

\begin{tabular}{|c|c|c|c|c|c|c|c|c|}
\hline Espèce végétale & Organe végétal & Utilisation & Recette & $\mathbf{A r}$ & Bo & Ko & Mo & Total citations \\
\hline Capparis sepiaria & Fruit & Amuse-gueule & $\operatorname{Rec} 20$ & 4 & 1 & 1 & 1 & 7 \\
\hline Capparis sepiaria & Fruit & Epice & $\operatorname{Rec} 21$ & & 1 & & & 1 \\
\hline Capparis sepiaria & Fruit & Légume & $\operatorname{Rec} 22$ & 3 & & & 1 & 4 \\
\hline Capparis sepiaria & Graine & Légume & $\operatorname{Rec} 23$ & 1 & & & & 1 \\
\hline Cassia occidentalis & Graine & Thé & $\operatorname{Rec} 24$ & & 1 & & & 1 \\
\hline Celtis integrifolia & Feuille & Légume & $\operatorname{Rec} 25$ & 8 & 2 & & 1 & 11 \\
\hline Celtis integrifolia & Fruit & Amuse-gueule & $\operatorname{Rec} 26$ & 1 & 2 & & & 3 \\
\hline Corchorus tridens & Feuille & Légume & $\operatorname{Rec} 27$ & 1 & 1 & & & 2 \\
\hline Crateva adansonii & Feuille & Amuse-gueule & $\operatorname{Rec} 28$ & 1 & & & & 1 \\
\hline Crateva adansonii & Feuille & Légume & $\operatorname{Rec} 29$ & 5 & & & 2 & 7 \\
\hline Crateva adansonii & Fruit & Amuse-gueule & $\operatorname{Rec} 30$ & 4 & & & 2 & 5 \\
\hline Diospyros mespiliformis & Feuille & Légume & $\operatorname{Rec} 31$ & & & 1 & & 1 \\
\hline Diospyros mespiliformis & Fruit & Amuse-gueule & $\operatorname{Rec} 32$ & 9 & 3 & 2 & 3 & 17 \\
\hline Echinochloa colona & Graine & Plat principal & $\operatorname{Rec} 33$ & 10 & 1 & 1 & 1 & 13 \\
\hline Ficus sycomorus & Feuille & Légume & $\operatorname{Rec} 34$ & & & 1 & & 1 \\
\hline Ficus sycomorus & Fruit & Amuse-gueule & $\operatorname{Rec} 35$ & & & 1 & & 1 \\
\hline Ficus platyphylla & Fruit & Amuse-gueule & $\operatorname{Rec} 36$ & 1 & & & & 1 \\
\hline Nympheae lotus & Bulbe & Plat principal & $\operatorname{Rec} 37$ & 3 & 3 & & & 6 \\
\hline Nympheae lotus & Fleur & Amuse-gueule & $\operatorname{Rec} 38$ & 1 & 1 & & & 2 \\
\hline Nymphaea lotus & Tige & Amuse-gueule & $\operatorname{Rec} 39$ & 1 & 1 & & & 2 \\
\hline Oryza barthii & Graine & Plat principal & $\operatorname{Rec} 40$ & 5 & & 1 & & 6 \\
\hline
\end{tabular}


J.L. BETTI et S.R. MEBERE YEMEFA'A / Int. J. Biol. Chem. Sci. 5(1): 291-303, 2011

\begin{tabular}{|c|c|c|c|c|c|c|c|c|}
\hline Espèce végétale & Organe végétal & Utilisation & Recette & $\mathbf{A r}$ & Bo & Ko & Mo & Total citations \\
\hline Panicum pansum & Graine & Plat principal & $\operatorname{Rec} 41$ & 10 & 1 & 1 & 1 & 13 \\
\hline Sclerocarya birrea & Fruit & Amuse-gueule & $\operatorname{Rec} 42$ & & & 1 & & 1 \\
\hline Tamarindus indica & Feuille & Epice & $\operatorname{Rec} 43$ & 5 & 2 & 3 & 1 & 11 \\
\hline Tamarindus indica & Feuille & Légume & $\operatorname{Rec} 44$ & & & & 3 & 3 \\
\hline Tamarindus indica & Fruit & Amuse-gueule & $\operatorname{Rec} 45$ & 1 & & 1 & 1 & 3 \\
\hline Tamarindus indica & Fruit & Epice & $\operatorname{Rec} 46$ & 10 & 3 & 2 & 2 & 17 \\
\hline Ziziphus mauritiana & Feuille & Légume & $\operatorname{Rec} 47$ & & & 1 & & 1 \\
\hline Ziziphus mauritiana & Fruit & Amuse-gueule & $\operatorname{Rec} 48$ & 9 & 3 & 2 & 2 & 16 \\
\hline Zizipus spina-christi & Fruit & Amuse-gueule & $\operatorname{Rec} 49$ & 6 & 2 & 1 & 1 & 10 \\
\hline Total & & & 49 & 161 & 38 & 35 & 34 & 268 \\
\hline
\end{tabular}
Groupes ethniques : $\mathrm{Ar}=$ Arabe choa $; \mathrm{Bo}=$ Bornoa $; \mathrm{Ko}=$ Kotoko $; \mathrm{Mo}=$ Mousgoum.

Tableau 2 : Liste des plantes alimentaires utilisées dans le parc national de Kalamaloué.

\begin{tabular}{lllc}
\hline Espèce & Nom arabe & Famille & Citations \\
\hline Acacia erythrocalyx Brenan & Echab & Mimosaceae & 4 \\
Acacia seyal Del. & Talla & Mimosaceae & 5 \\
Acacia sieberiana DC. & Kouk & Mimosaceae & 2 \\
Adansonia digitata L. & Baké & Bombacaceae & 10 \\
Balanites aegyptiaca (L.) Del. & Idilit & Zygophyllaceae & 43 \\
Boscia senegalensis (Pers.) Lam. Ex Poir. & Mourhet & Capparaceae & 20
\end{tabular}


J.L. BETTI et S.R. MEBERE YEMEFA'A / Int. J. Biol. Chem. Sci. 5(1): 291-303, 2011

\begin{tabular}{|c|c|c|c|}
\hline Espèce & Nom arabe & Famille & Citations \\
\hline Cadaba farinosa Forsk. & Sireh & Capparaceae & 1 \\
\hline Calotropis procera (Ait.) Ait. F. & Echoro & Asclepiadaceae & 2 \\
\hline Capparis fascicularis D.C. & Gouloum & Capparaceae & 14 \\
\hline Capparis sepiaria $\mathrm{L}$. & Gouloum & Capparaceae & 14 \\
\hline Cassia occidentalis L. & Enkalaoua & Caesalpiniaceae & 1 \\
\hline Celtis toka (Forssk.) Hepper \& Wood (Syn.: Celtis integrifolia Lam.) & Ngouso & Ulmaceae & 14 \\
\hline Corchorus tridens L. & Moulohié & Tiliaceae & 2 \\
\hline Crateva adansonii DC. & Dapkar & Capparaceae & 14 \\
\hline Diospyros mespiliformis Hochst. ex A. DC. & Djogan & Ebenaceae & 18 \\
\hline Echinochloa colona & Kereb & Poaceae & 13 \\
\hline $\begin{array}{l}\text { Ficus sycomorus L. subsp. gnaphalocarpa (Miq.) C.C. Berg (Syn.: F. } \\
\text { gnaphalocarpa) }\end{array}$ & Dimeze & Moraceae & 3 \\
\hline Ficus platyphylla Delile & Djakdjak & Moraceae & 1 \\
\hline Nymphaea lotus L. & Noar & Nymphaeaceae & 6 \\
\hline Oryza barthii & Riss & Oryzaceae & 6 \\
\hline Panicum pansum & Kereb & Poaceae & 13 \\
\hline Sclerocarya birrea (A. Rich.) Hochst. & & Anacardiaceae & 1 \\
\hline Tamarindus indica $\mathrm{L}$. & Ardep & Caesalpiniaceae & 34 \\
\hline Ziziphus mauritiana Lam. & Nabak & Rhamnaceae & 17 \\
\hline Zizipus spina-christi (L.) Desf. var. spina-christi & Mardo & Rhamnaceae & 10 \\
\hline Total & & & 268 \\
\hline
\end{tabular}




\section{DISCUSSION}

De nombreuses convergences d'emplois des recettes ont été identifiées entre les différents groupes ethniques enquêtés. Les recettes les plus citées sont: la consommation des fruits de Balanites aegyptiaca (21 citations), Diospyros mespiliformis (17), ou encore de Ziziphus mauritiana (17) comme amuse-gueule ou fruit de bouche, et aussi la consommation du fruit de Tamarindus indica comme épice (16). Ces recettes sont signalées dans tous les groupes ethniques. Dans l'ensemble, douze recettes sont indiquées dans tous les quatre groupes ethniques rencontrés. Les Arabes choa partagent 55,2\% de leurs recettes avec tous les autres groupes. Ils partagent 21 recettes avec les Bornoa, 14 avec les Kotoko et 19 avec les Mousgoum. Les Bornoa ont cité 23 recettes dont deux seulement leur sont propres à savoir la consommation des fruits de Capparis sepiaria comme épices et la prise des graines de Cassia occidentalis sous forme de thé. Les Kotoko ont cité 23 recettes, 6 de ces recettes leurs sont propres et ils partagent les 17 autres avec les autres groupes ethniques. Les Mousgoum ont cité 21 recettes, et aucune de ces recettes ne leur est unique ou propre. Vingt six (25) recettes (soit 51\%) sont citées dans un seul groupe ethnique.

La famille des Capparaceae est la plus représentée avec 5 espèces et 63 citations. Sur le plan écologique, les Capparaceae se développent bien dans des zones Soudanosahéliennes (Aubreville, 1950). Il s'agit donc d'une famille normalement très présente dans le parc national de Kalamaloué.

L'importance des amuse-gueule s'explique par le fait qu'en plus du complément alimentaire, ils représentent une source complémentaire de revenu pour les populations locales. En effet, les populations de Kalamaloué affirment vendre tous les produits utilisés comme amuse-gueule, à l'exception de Nymphaea lotus et de cassia occidentalis. La gomme arabique de Acacia seyal est de qualité presque égale à celle de $A$. senegal qui est très prisée sur le marché international (Arbonnier, 2000). Les gommes contiennent des polysaccharides et de petites quantités de protéines et des sels minéraux. La gomme arabique se présente comme un exsudat sec obtenu à partir des branches et tiges de Acacia seyal (FAO, 1995b, 1998). L'usage des feuilles et fruits de Adansonia digitata, Balanites aegyptiaca et Tamarindus indica dans l'alimentation a déjà été signalé au Cameroun (Eyog Matig et al., 2006). Balanites aegyptiaca et Ziziphus mauritiana sont listées parmi les fruits sauvages comestibles vendus sur les marchés de Bangui en République Centrafricaine. La pulpe des fruits des deux espèces est consommée fraîche ou sèche. On réduit aussi parfois les fruits de Z. mauritiana en farine pour servir à la cuisson des aliments (Apema et al., 2010). Les graines séchées de Balanites aegyptiaca encore appelé Kournaka en langue arabe fournissent selon Aubreville (1950), une huile très appréciée par les communautés Tchadiennes. Burkill (2000) signale que les fruits de Sclerocarya birrea donnent une boisson très alcoolisée, appréciée en Afrique de l'Ouest. Arbonier (2000) révèle que les fruits de Tamarindus indica fournissent une boisson rafraîchissante et acidulée riche en vitamine $\mathrm{C}$, très consommée et commercialisée en Afrique de l'Ouest. Ces autres formes d'utilisations des amuse-gueule peuvent, si elles sont adoptées, contribuer à la réduction de la pauvreté des populations et implicitement, réduire les conflits entre les populations riveraines du parc national de Kalamaloué et l'administration en charge de la conservation dudit parc sur la gestion des ressources naturelles. Les fruits sauvages contiennent les nutriments vitaux (hydrates de carbones, protéines, et les minéraux) et les vitamines essentiels qui sont très importants pour la croissance des enfants qui sont souvent astreints à la malnutrition et aux maladies connexes. En conditions d'extrême 
aridité, les fruitiers sauvages telles que Boscia senegalensis et Ziziphus sp jouent un rôle important dans la sécurité alimentaire. Les fruitiers sauvages de Kalamaloué qui possèdent une valeur commerciale réelle en Afrique sont entre autres Tamarindus indica, Ziziphus mauritiana, Adansonia digitata, Sclerocarya birrea (Chikamai and Tchatat, 2004).

Les légumes sauvages, tout comme les autres formes de consommation des plantes alimentaires sauvages, jouent un rôle important dans le bien-être des communautés rurales des pays en voie de développement. Ils remplacent facilement les légumes domestiques en période de disette (Asfaw and Tadesse, 2001). Les légumes sauvages constituent non seulement des compléments nutritionnels, très indispensable à l'équilibre alimentaire (Somnasang and Moremo-Black, 2000), mais aussi et surtout des sources alternatives des revenus pour les communautés très démunies (Melnyk, 1996). Burkill (2000) signale la consommation des feuilles de Corchorus tridens par les populations Tiv du Nigéria et Tenda du Sénégal. Les Soninké du Sénégal consomment largement les feuilles de Adansonia digitata (Chastanet, 1991). En Afrique de l'Ouest, les feuilles et fruits de Capparis sepiaria sont utilisés comme légume en période de disette. L'apport nutritionnel des légumes est surtout qualitatif. Les feuilles de Adansonia digitata contiennent des vitamines A, B2 et C, ainsi que du calcium et acide nicotinique. Les fruits et feuilles de A. digitata utilisés comme plat d'accompagnement ont une faible valeur énergétique mais apportent des compléments indispensables aux calories (protéines, vitamines, sels minéraux). Dans le contexte de pauvreté caractérisant les zones rurales, on peut dire que ces plantes jouent un rôle important dans l'équilibre du régime alimentaire, en remédiant à la consommation irrégulière des produits animaux (Chastanet, 1991).
Les peuples de l'Afrique de l'Ouest utilisent les feuilles de Capparis fascicularis comme épice de sauce (Arbonnier, 2000). Dans la région de Borno au Nigéria, c'est Capparis sepiaria qui est plutôt utilisée (Burkill, 2000).

$\mathrm{Au}$ cours de l'année 2004, le département du Logone et Chari a été l'une des localités de l'Extrême-Nord qui a été particulièrement touchée par la famine. Plusieurs régions en Afrique disposent chacune des aliments de disette. Ainsi par exemple, le Nigeria, le Niger, le Soudan considèrent Boscia senegalensis, Capparis sepiaria, et Guiera senegalensis comme aliments de disette (Arbonnier, 2000 ; Burkill, 2000 ; Okpo et al., 2001). Chastanet (1991) relève que les Soninké du Sénégal consomment largement le fonio sauvage ou Panicum sp., comme substitut du mil ou du maïs pendant les périodes de soudures.

\section{Conclusion}

L'étude réalisée dans les villages riverains du parc national de Kalamaloué avait pour but de faire une analyse des usages traditionnels des plantes alimentaires récoltées dans le parc. Un total de 27 espèces végétales a été signalé comme plantes alimentaires. Ces espèces interviennent dans 50 recettes alimentaires différentes. Les fruits, graines et feuilles sont les organes végétaux les plus cités, tandis que les amuse-gueule sont les modes de consommation des plantes les plus mentionnés. De nombreuses convergences d'usage des recettes sont relevées entre les différents groupes ethniques. Les espèces les plus citées sont Balanites aegyptiaca ou dattier du désert, Tamarindus indica ou tamarinier, Boscia senegalensis, Diospyros mespiliformis ou ébène de savane, et Ziziphus mauritiana. Ces espèces sont également mentionnées pour les mêmes usages dans d'autres pays africains. Il existe également de nombreuses recettes, qui ne sont signalées que dans un seul groupe ethnique. Près de la 
moitié des recettes recensées ont des usages limitées dans un seul groupe ethnique. La plupart de ces recettes peuvent constituer l'originalité de ce travail. Les résultats présentés ici peuvent permettre d'amorcer les processus de valorisation des plantes alimentaires comme alternative au braconnage. L'administration en charge des aires protégées devraient tenir compte de ces résultats qui montrent l'importance des PFNL pour les populations locales lors de l'élaboration du plan d'aménagement de Kalamaloué. La mise en place d'un bon système de gestion participative passe par une meilleure prise en compte des relations homme - ressources du parc.

\section{REMERCIEMENTS}

Ce travail a été rendu possible grâce au soutien logistique de l'Ecole de Faune de Garoua.

\section{REFERENCES}

Apema R, Mozouloua D, Madiapevo SN. 2010. Inventaire préliminaire des fruits sauvages comestibles vendus sur les marchés de Bangui. In Systématique et conservation des Plantes Africaines, van der Burgt X, van der Maesen J, Onana JM (eds). Royal Botanic Garden : Kew; 313319.

Arbonnier. 2000. Arbres, Arbustes et Lianes des Zones Sèches d'Afrique de l'Ouest. CIRAD, MNHN, UICN : Montpellier, France.

Asfaw Z, Tadesse M. 2001. Prospects for sustainable use and development of wild food plants in Ethiopia. Economic Botany 55: 47-62.

Aubreville 1950. Flore Forestière SoudanoGuinéenne. Société d'Edition Géographique, Maritime et Coloniales : Paris, France.

Burkill HM. 2000. The Useful Plants of West Tropical Africa (vol 5). Families s - w. Royal Botanic Garden: Kew.
Chastanet M. 1991. La cueillette des plantes alimentaires en pays Soninké, Sénégal depuis la fin du XIXème siècle. Histoire et devenir d'un savoir-faire. In Savoirs Paysans et Développement, Duprès (ed). Karthala Orstom: Paris, France ; 253-287.

Chikamai B, Tchatat M. 2004. Forest management for non wood forest products and services in Africa. A report prepared for the project, "Lessons learnt on sustainable forest management in Africa." KSLA/AFORNET/FAO, p. 35.

Eyog Matig O, Ndoye O, Kengue J, Awono A. 2006. Les Fruitiers Forestiers Comestibles $d u$ Cameroun. International Plant Genetic Resources Institute; 204.

FAO. 1995a. Non-wood forest products for rural income and sustainable forestry. Non-Wood Forest Products series 7. Rome.

FAO 1995b. Gums, resins and latexes of plant origin. Non-Wood Forest Products No. 6, Rome.

FAO 1998. Food and Nutrition Paper No. 52. FAO, Rome.

Letouzey R. 1968. Etude Phytogéographique $d u$ Cameroun. Editions P. Lechevalier: Paris ; 511.

Melnyk M. 1996. Indigenous enterprise for the domestication of trees and the commercialization of their fruits. In Domestication and Commercialization of Non Timber Forest Products in Agroforestry Systems, Leakey RRB, Temu AB, Melnyk M, Vantomme P (eds). Non wood Forest Products No. 9 FAO: Rome, Italy; 97-103.

MINEF 1996. A Compendium of official instruments on forest and wildlife management in Cameroon. Imprimerie Nationale, Yaoundé, p.185.

MINEF 1994. Law No. 94/01 of 20 January 1994 to lay down Forestry, Wildlife and Fisheries regulations, Imprimerie Nationale, Yaoundé, p. 43. 
Ndoye O, Ruiz-Perez M, Eyebe A. 1997. The market of non-timber forest products in the humid forest zone of Cameroon. Rural Develoment Forestry Network Paper 22c. ODI. London, Royaume-Uni.

Okpo SO, Fatokun F, Adeyeni OO. 2001. Analgesic and anti-inflammatory activity of Crinum glaucum acqueous extract. Journal of Ethnopharmacology, 78(2): 207-211.

Somnasang P, Moreno-Black G. 2000. Knowing, gathering and eating: knowledge and attitudes about wild food in an Isan village in Northeastern Thailand. Journal of Ethnobiology, 20: 197-216.
Souane Thirakoul B. 1990. Manuel de dendrologie des savanes boisées. Agence Canadienne de développement International. Projet Appui Institutionnel Phase 2.

Tieguhong Chupezi J, Betti JL. 2008. Forest and Protected Areas in Cameroon: Progress has been significant but challenges remain. International Tropical Timber Organization (ITTO), Tropical Forest Update, 18/1: 6-9.

Tersiguel P, Becker C. 1997. Développement durable au sahel. Karthala \& Société espace, temps. Paris, France. 